\title{
FERTILIZANTES NA PRODUÇÃO DE MUDAS DE OLIVEIRA ‘ARBEQUINA’
}

\section{FERTILIZERS ON PRODUCTION OF OLIVE ROOTED STEM CUTTINGS 'ARBEQUINA'}

\author{
João VIEIRA NETO ${ }^{1,5}$ \\ Geraldo Magela de Almeida CANÇADO ${ }^{2}$ \\ Adelson Francisco de OLIVIERA ${ }^{3}$ \\ Hugo Adelande de MESQUITA ${ }^{3}$ \\ Alessandro Dal'col LÚCIO ${ }^{4}$ \\ Luiz Fernando de Oliveira da SILVA ${ }^{1}$
}

\begin{abstract}
RESUMO
O trabalho teve por objetivo avaliar os efeitos da aplicação de fertilizantes comerciais na fase final de produção de mudas de oliveira. A pesquisa foi conduzida na Fazenda da EPAMIG, localizada no município de Maria da Fé, MG. As atividades de pesquisa iniciaram em 2008, sendo utilizadas estacas semilenhosas enraizadas de 'Arbequina'. O delineamento experimental utilizado foi o inteiramente casualizado, com quatro repetições e 12 mudas por parcela, cultivadas em sacolas plásticas com capacidade de $2 \mathrm{dm}^{3}$ de substrato. Avaliou-se quatro fertilizantes comerciais: Ativo ${ }^{\circledR}$; Brotax-Micro ${ }^{\circledR}$; Fert Bokashi $^{\circledR}$; e Nippoterra ${ }^{\circledR}$, em cinco dosagens $\left(\mathrm{cm}^{3} \mathrm{dm}^{-3}\right): 0 ; 1 ; 2 ; 3$; e 4. Os fertilizantes foram aplicados em intervalos de 15 dias após instalação do ensaio, durante um período de 120 dias. Cada parcela experimental foi pulverizada com $0,5 \mathrm{dm}^{3} \mathrm{de}$ solução. Aos 60 dias foram avaliados o número médio de brotações e o comprimento médio de brotações e aos 120 dias, altura da planta; número médio de raízes; comprimento do sistema radicular; massa da fitomassa seca da parte aérea e massa da fitomassa seca da raiz. Observou-se pelos resultados que os fertilizantes Ativo ${ }^{\circledR}$, Brotax-Micro ${ }^{\circledR}$, Fert Bokashi ${ }^{\circledR}$ e Nippoterra ${ }^{\circledR}$ tiveram desempenho favorável na etapa final de produção de mudas de oliveira; Todos fertilizantes testados, quando aplicados na dosagem de $4 \mathrm{~cm}^{3} \mathrm{dm}^{-3}$, proporcionaram mudas maiores e com sistema radicular mais desenvolvido, em relação à testemunha.
\end{abstract}

Palavras-chave: Propagação; olivicultura; nutrição mineral; adubação.

\section{ABSTRACT}

In this work evaluated the effect of fertilizers in the final phase of olive rooted stem cuttings production. The experimental set was carried out in the Experimental Farm of EPAMIG, located in the city of Maria da Fé, Minas Gerais State, Brazil. The work developed with rooted hard-cuttings of Arbequina cultivar started in beginning of February 2008 . The experimental design was of completely randomized plots with four replicates of 12 plants for each experimental plot. The plants were cultivated in pots containing $2 \mathrm{dm}^{3}$ of soil substratum. Four commercial fertilizers were evaluated: Ativo ${ }^{\circledR}$; BrotaxMicro $^{\circledR}$; Fert Bokashi ${ }^{\circledR}$; and Nippoterra ${ }^{\circledR}$, in five concentration levels $\left(\mathrm{cm}^{3} \mathrm{dm}^{-3}\right): 0 ; 1 ; 2 ; 3$; and 4. All fertilizer treatments were applied at 15-days intervals during the period of 120 days and each experimental plot was sprayed with $0,5 \mathrm{dm}^{3}$ of treatment solution. After 60 days the average number of buds and bud length were evaluated. Further, after 120 days of treatment, were evaluated the averages of shoot and root length; root number; shoot fresh and dry weight; and root fresh and dry weight. During the last phase of olive rooted cuttings production, the treatments using the fertilizers Ativo ${ }^{\circledR}$, Brotax-Micro $^{\circledR}$, Fert Bokashi $^{\circledR}$ and Nippoterra ${ }^{\circledR}$ showed better result than the treatment control (no fertilizer). The fertilizers with the best performance were Brotax-Micro ${ }^{\circledR}$ and Nippoterra ${ }^{\circledR}$ while the dosage of $4 \mathrm{~cm}^{3} \mathrm{dm}^{-3}$ showed the higher response for all fertilizers tested.

Key-words: Propagation; oliviculture; mineral nutrition; fertilization.

\footnotetext{
1 Engenheiro Agrônomo, D. Sc. Empresa de Pesquisa Agropecuária de Minas Gerais - Fazenda Experimental de Maria da Fé. Bairro Vargedo, 35517-000, Maria da Fé, Minas Gerais, Brasil. E-mail: joaovieira@epamig.br; luizfernando.agronomia@gmail.com. Bolsistas da Fundação de Amparo à Pesquisa do Estado de Minas Gerais (FAPEMIG)

2 Engenheiro Agrônomo, D. Sc. Empresa de Pesquisa Agropecuária de Minas Gerais - Fazenda Experimental de Caldas. Caldas, Minas Gerais, Brasil. E-mail: cancado@epamig.br. Bolsista da Fundação de Amparo à Pesquisa do Estado de Minas Gerais (FAPEMIG)

3 Engenheiro Agrônomo, D. Sc. Empresa de Pesquisa Agropecuária de Minas Gerais. Fazenda Experimental de Lavras. Lavras, Minas Gerais, Brasil. E-mail: adelson@epamig.br; adelande@epamig.br Bolsistas da Fundação de Amparo à Pesquisa do Estado de Minas Gerais (FAPEMIG).

Engenheiro Agrônomo, D. Sc. Universidade Federal de Santa Maria. Santa Maria. Camobí, Rio Grande do Sul, Brasil. E-mail: adlucio@smail.ufsm.br.

5 . Autor para correspondência
}

Scientia Agraria, Curitiba, v.11, n.1, p.049-055, J an./F eb. 2010. 
VIEIRA NETO, J. et al. Fertilizantes na produção de mudas...

\section{INTRODUÇÃO}

A oliveira, Olea europaea L. (Oleaceae), teve seu cultivo iniciado a quatro mil anos a.C. próximo ao Mar Morto, região que abrange a Síria, Líbano e Israel, expandindo-se para o Ocidente pela bacia do Mediterrâneo. Atualmente é cultivada em todos os continentes, mas sua produção comercial está concentrada em países da União Européia sendo essa região, de acordo com Rallo (2005), a maior produtora mundial. A importância dessa atividade agrícola relaciona-se principalmente com a elaboração de azeite (Aued-Pimentel, 2008; Oliveira et al., 2009a).

$\mathrm{Na}$ região mediterrânea, em países da Comunidade Econômica Européia, são produzidos $79,8 \%$ do azeite de oliva mundial, destaque para a Espanha (42,9\%), Itália (17,5\%) e Grécia (12,2\%), além de responder por quase $80 \%$ das exportações mundiais (Mesquita et al., 2006).

O cultivo comercial no Brasil é uma atividade agrícola recente e em expansão (Oliveira et al., 2009a). Tendo como base o volume de 214 mil $t$ de azeitona importadas em 2008, CONAB (2009), estima-se um mercado potencial de mudas de aproximadamente 11 milhões de unidades, exigindo da pesquisa informações técnicas para sua produção.

A propagação assexuada realizada por métodos de enxertia e estaquia, além de oferecer maior possibilidade de uniformidade de plantas produtivas no pomar, devido à perpetuação de características desejáveis e início precoce da produção, também pode resolver importantes problemas fitossanitários (São José et al., 1994; Natale et al., 2002; Souza \& Lima, 2005).

O sistema de multiplicação da oliveira, empregado nos últimos vinte anos na maioria das regiões produtoras, resume-se em três fases bem distintas. O enraizamento, durante o qual se tem a emissão de raízes adventícias na base das estacas; a aclimatação, onde se promove o funcionamento do sistema radicular obtido na fase anterior, e finalmente, a formação das mudas em viveiro (Oliveira, 2007; Soares et al.,2007).

Após o enraizamento adequado das estacas, a última fase na multiplicação da oliveira é a obtenção da muda para o plantio. O tamanho padrão é de 80 a $100 \mathrm{~cm}$ de altura com uma única haste principal, iniciando-se a formação da copa da planta com até quatro ramos ou pernadas. Essa condição se dá preferentemente em viveiros devidamente manejados, com uso de irrigação, fornecimento de nutrientes e o controle preventivo de pragas e doenças (Caballero \& Del Rio, 1998; Oliveira et al., 2003; Oliveira et al., 2009b).

A formação da muda no menor tempo e com o máximo de vigor depende das características do substrato utilizado e do manejo em viveiro. A definição desses fatores é preponderante no processo de produção de mudas de qualquer espécie vegetal (Fachinello et al., 2005).

No Brasil existe carência de tecnologia aplicada ao cultivo da oliveira, sendo importante estudar entre outros aspectos da cultura, o manejo da fertilidade e nutrição da planta, desde a fase de muda até a produção a campo. Segundo Larcher (2000), os principais nutrientes necessários ao desenvolvimento da planta devem estar prontamente disponíveis desde os estágios iniciais de crescimento, promovendo assim o máximo desenvolvimento, evitando que ocorram desequilíbrios entre 0 conteúdo mineral e o incremento de fitomassa seca.

A disponibilidade de mudas vigorosas e sadias de oliveira é considerada como um fator chave no estabelecimento e produção de pomares comerciais. Dessa forma, o presente trabalho teve por objetivo avaliar os efeitos da aplicação de diferentes fertilizantes comerciais na fase final de produção de mudas de oliveira.

\section{MATERIAL E MÉTODOS}

A pesquisa foi conduzida em viveiro na Fazenda da EPAMIG, localizada no município de Maria da Fé, micro região da serra da Mantiqueira, região sul de Minas Gerais. O viveiro era coberto com sombrite $(50 \%$ de sombreamento), com irrigação por microasperssores distribuídos regularmente na parte superior do mesmo.

O trabalho teve início no dia 01 de fevereiro de 2008, durante a estação de crescimento da cultura (verão/outono). Para instalação do experimento, utilizou-se estacas semilenhosas da cultivar Arbequina, pertencente ao Banco de Germoplasma da EPAMIG, em Maria da Fé. As estacas tinham cerca $12 \mathrm{~cm}$ de comprimento, com dois pares de folhas devidamente enraizadas em perlita, aclimatadas e as mudas padronizadas quanto ao tamanho, com cerca de $20 \mathrm{~cm}$.

$O$ delineamento experimental utilizado foi o inteiramente casualizado com parcelas subdivididas, com quatro repetições de 12 mudas por parcela, sendo a parcela útil composta pelas três plantas centrais, cultivadas em sacolas plásticas com capacidade de $2 \mathrm{dm}^{3}$ de substrato. Foram avaliados dois fatores, sendo quatro fertilizantes (Ativo $^{\circledR}$; Brotax-Micro ${ }^{\circledR}$; Fert Bokashi ${ }^{\circledR}$; e Nippoterra ${ }^{\circledR}$ ) nas parcelas principais, e cinco dosagens $(0 ; 1 ; 2 ; 3$; e $4 \mathrm{~cm}^{3} \mathrm{dm}^{-3}$ ) nas subparcelas.

A composição das formulações e as garantias asseguradas pelos fabricantes dos fertilizantes são descritas na Tabela 1.

O substrato utilizado foi constituído por terra de subsolo e composto orgânico comercial (Provaso ${ }^{\circledR}$ ) na proporção de $1: 1(\mathrm{v} / \mathrm{v})$, enriquecido com $5 \mathrm{~kg}$ de superfosfato simples (18 dag kg-1 de $\mathrm{P}_{2} \mathrm{O}_{5}$ ), $1 \mathrm{~kg}$ de cloreto de potássio (58 dag kg ${ }^{-1} \mathrm{de}$ $\mathrm{K}_{2} \mathrm{O}$ ) e $2,5 \mathrm{~kg}$ de calcário dolomítico (PRNT 100\%) para cada $1 \mathrm{~m}^{3}$ de substrato. $\mathrm{O}$ composto orgânico comercial Provaso ${ }^{\circledR}$ possuia na sua constituição as quantidades de, no mínimo, 1,37 dag $\mathrm{kg}^{-1}$ de $\mathrm{N}$, 1,65 dag kg ${ }^{-1}$ de $\mathrm{P}(\mathrm{Pt}), 0,92$ dag $\mathrm{kg}^{-1}$ de $\mathrm{K}\left(\mathrm{K}_{2} \mathrm{O}\right), 4$ dag $\mathrm{kg}^{-1}$ de $\mathrm{Ca}, 0,34$ de S, 0,1 dag $\mathrm{kg}^{-1}$ de $\mathrm{Na}, 1,35$ dag $\mathrm{kg}^{-1}$ de Fe, 0,6 dag kg ${ }^{-1}$ de $\mathrm{Mg}, 0,06$ dag $\mathrm{kg}^{-1}$ de Mn, 0,06 dag kg-1 de Zn, 0,02 dag kg-1 de Cu, 0,02 dag $\mathrm{kg}^{-1}$ de $\mathrm{B}$, umidade 47,4 dag $\mathrm{kg}^{-1}, \mathrm{pH} 7,2$, CTC 
VIEIRA NETO, J. et al. Fertilizantes na produção de mudas...

TABELA 1 - Fertilizantes e garantias mínimas de composição asseguradas pelos fabricantes. EPAMIG, Maria da Fé - MG, 2009.

\begin{tabular}{ccccc}
\hline Garantias (dag kg $\left.^{-1}\right)$ & Ativo $^{(\Theta)}$ & Brotax-Micro $^{(\Theta}$ & Fert Bokashi $^{(\Theta}$ & Nippoterra $^{(\Theta)}$ \\
\hline $\mathrm{N}$ & - & 7 & 1,00 & 8,00 \\
$\mathrm{P}_{2} \mathrm{O}_{5}$ & - & 6 & - & 6,00 \\
$\mathrm{~K}_{2} \mathrm{O}$ & - & 7 & - & 15,00 \\
$\mathrm{Zn}$ & 1,00 & 0,05 & - & 0,20 \\
$\mathrm{Cu}$ & 0,30 & 0,05 & - & 0,05 \\
$\mathrm{Fe}$ & 0,50 & 0,20 & - & 0,20 \\
$\mathrm{Mn}$ & 0,50 & 0,50 & - & 0,50 \\
$\mathrm{~B}$ & 0,50 & 0,50 & - & 0,40 \\
$\mathrm{Mo}$ & 0,10 & 0,05 & - & 0,05 \\
$\mathrm{~S}$ & 2,00 & - & - & - \\
$\mathrm{Mg}$ & 1,00 & 0,20 & - & - \\
$\mathrm{Ca}$ & - & 0,10 & 8,00 & - \\
$\mathrm{C}$ & - & - & -
\end{tabular}

${ }^{1}$ Carbono orgânico

$250 \mathrm{cmol}_{\mathrm{c}} \mathrm{dm}^{-3}$, relação CTC/C 1,1, carbono orgânico 22 dag kg-1 e relação $\mathrm{C} / \mathrm{N} 17 / 1$.

Os fertilizantes foram aplicados, com 0,5 $\mathrm{dm}^{3}$ de solução em cada parcela experimental, nas plantas, com auxílio de um pulverizador costal. As aplicações foram realizadas em intervalos de 15 dias após a instalação do experimento, durante um período total de 120 dias.

Após 60 dias da instalação do experimento, foram avaliados o número e o comprimento médio das brotações. Aos 120 dias, após a última aplicação, foram avaliadas as seguintes características: altura da planta (m); número médio de raízes; comprimento do sistema radicular $(\mathrm{cm})$; massa seca da parte aérea e da raiz (g planta $\left.{ }^{-1}\right)$.

A análise estatística foi realizada via análise de variância com teste $\mathrm{F}$ e, caso efeitos significativos dos fatores em avaliação, foram aplicados os procedimentos complementares (análise de regressão). Em todas as análises adotou-se $5 \%$ de probabilidade de erro, tendo sido utilizado o programa estatístico NTIA (EMBRAPA, 1997).

\section{RESULTADOS E DISCUSSÃO}

Observou-se pelos resultados da análise de variância que todas as variáveis apresentaram interação significativa entre fertilizantes e dosagens. Ao realizar-se o desdobramento das dosagens dentro de cada fertilizante, verificou-se que o número médio de brotações e o comprimento médio de brotações das mudas de oliveira aos 60 dias aumentaram linearmente em resposta as doses dos fertilizantes Brotax-Micro ${ }^{\circledR}$ (Figura 1A) e Fert Bokashi $^{\circledR}$ (Figura 1B), respectivamente. O maior número de brotações foi obtido quando utilizou-se o fertilizante Brotax-Micro ${ }^{\circledR}$ a $4 \mathrm{~cm}^{3} \mathrm{dm}^{-3}(1,66 \mathrm{~cm})$. Em relação ao comprimento médio das brotações, o melhor resultado foi obtido ao utilizar-se o fertilizante Ferti Bokashi ${ }^{\circledR}$ a $4 \mathrm{~cm}^{3} \mathrm{dm}^{-3}(5,51 \mathrm{~cm})$. Para os demais fertilizantes testados, 0 incremento nas doses não promoveu aumento significativo nessas variáveis.

A relação mais equilibrada entre $\mathrm{N}$ e $\mathrm{K}$, nesses fertilizantes, pode ter contribuído para esse resultado. No caso do Brotax-Micro ${ }^{\circledR}$ a relação N/K é de $(1,0)$, próxima a apresenta por Malavolta (2006), $(1 / 3)$, em contraposição à verificada no Nippoterra ${ }^{\circledR}$ $(0,53)$.

Efeito linear foi encontrado também para Nippoterra ${ }^{\circledR}$ em relação à altura da planta e ao número médio de raízes (Figuras $2 \mathrm{~A}$ e $2 \mathrm{~B}$ ) aos 120 dias. De acordo com as equações ajustadas, esse fertilizante proporcionou um incremento de $44,8 \%$ na altura da muda, alcançando $48,5 \mathrm{~cm}$ aos 120 dias, e incremento de $19,4 \%$ no número de raízes, em relação à testemunha. Não houve efeito dos fertilizantes Brotax-Micro ${ }^{\circledR}$, Ativo ${ }^{\circledR}$ e Fert Bokashi ${ }^{\circledR}$ para altura da planta e número médio de raízes.

Em trabalho realizado com mudas de cupuaçuzeiro, Fernandes et al. (2003) verificaram efeito linear do fósforo e do zinco sobre 0 crescimento das plantas, em altura. Esses elementos estão presentes nos fertilizantes Nippoterra ${ }^{\circledR}$ e Brotax-Micro ${ }^{\circledR}$, no entanto, o primeiro apresenta quatro vezes mais $\mathrm{Zn}\left(0,20\right.$ dag $\left.\mathrm{kg}^{-1}\right)$ do que 0 último $\left(0,05 \mathrm{dag} \mathrm{kg}^{-1}\right)$, com praticamente a mesma quantidade de $\mathrm{P}_{2} \mathrm{O}_{5}$ (8 dag $\left.\mathrm{kg}^{-1}\right)$. A proporção entre esses elementos próxima à verificada por Fernandes et al. (2003) no Nippoterra ${ }^{\circledR}$ poderia justificar o melhor resultado desse fertilizante em relação ao Brotax-Micro ${ }^{\circledR}$. 
VIEIRA NETO, J. et al. Fertilizantes na produção de mudas...
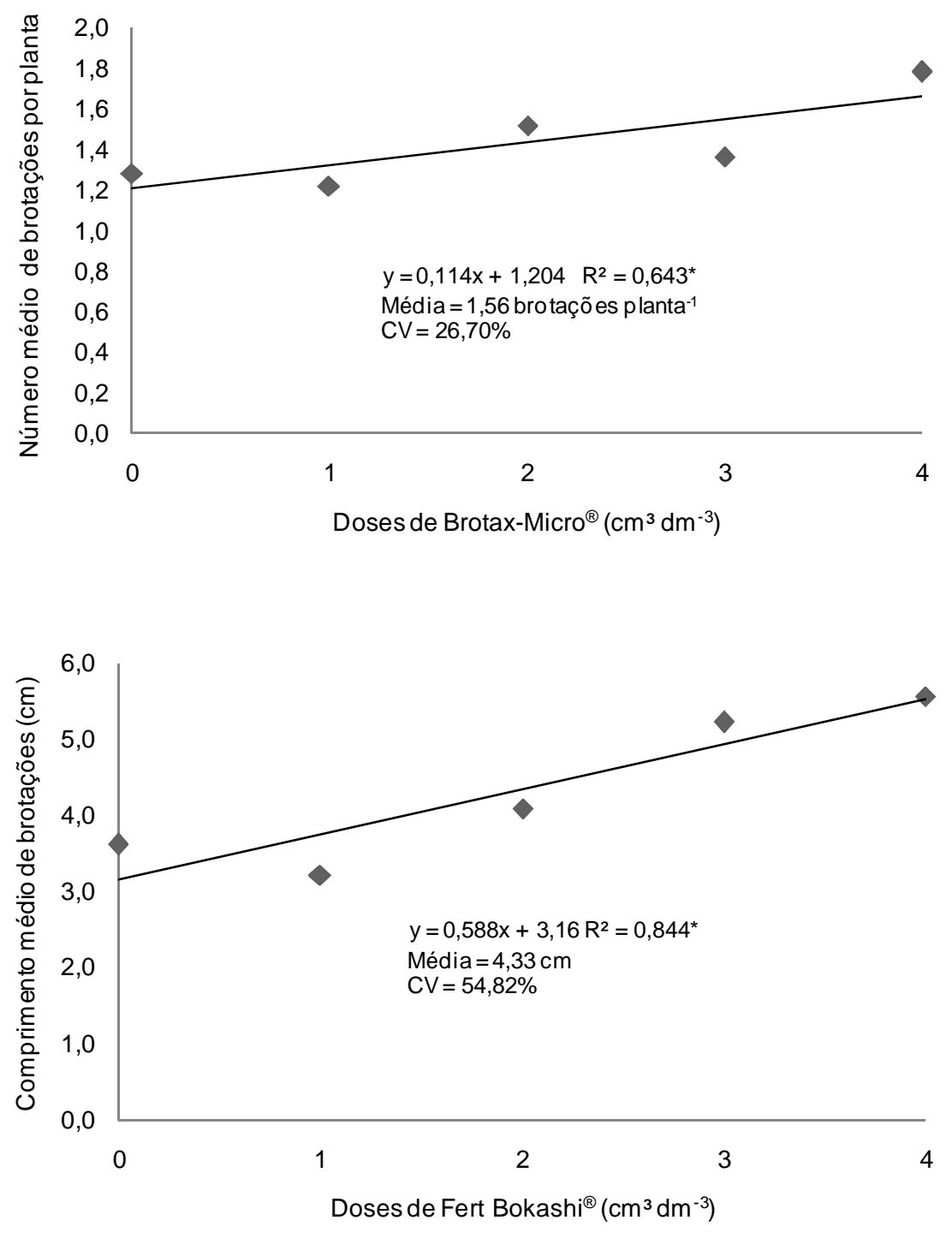

FIGURA 1 - Número médio de brotações por planta (A) e comprimento médio de brotações de mudas (B) da oliveira 'Arbequina', após 60 dias da aplicação de diferentes doses de Brotax-Micro ${ }^{\circledR}$ e de Fert Bokashi $^{\circledR}$, respectivamente. EPAMIG, Maria da Fé - MG, 2009. * Significativo a 5\% de probabilidade pelo teste $\mathrm{F}$.

Segundo Vieira Neto et al. (2009) a dosagem de $1 \mathrm{~cm}^{3} \mathrm{dm}^{-3}$ de Nippoterra ${ }^{\circledR}$, aplicada na fase final de produção de mudas de oliveira das cultivares Arbequina, Ascolano 315 e Grappolo 541, proporcionou resposta positiva para a altura de plantas, com média de $29,3 \mathrm{~cm}$ de altura aos 120 dias em viveiro, contra $22,1 \mathrm{~cm}$ da testemunha. Esse resultado está em concordância com os resultados obtidos nesse estudo e corrobora com efeito positivo das dosagens desse fertilizante no desenvolvimento das mudas de oliveira.

O comprimento do sistema radicular apresentou comportamento quadrático com as doses de fertilizantes Brotax-Micro ${ }^{\circledR}$ e Nippoterra ${ }^{\circledR}$ (Figura 3), apresentando o máximo comprimento estimado quando se utilizou $2,46 \mathrm{~cm}^{3} \mathrm{dm}^{-3}$ de Brotax-Micro $^{\circledR}$, atingindo $45,22 \mathrm{~cm}$ e quando tratadas com 2,04 $\mathrm{cm}^{3} \mathrm{dm}^{-3}$ de Nippoterra ${ }^{\circledR}(38,49$ $\mathrm{cm})$, diminuído a partir daí. Resultado semelhante foi encontrado por Vieira Neto et al. (2009), em ensaio com mudas de oliveira. Esse resultado se deve ao fato dos fertilizantes Ativo ${ }^{\circledR}$ e Fert Bokashi ${ }^{\circledR}$ não apresentarem teores de fósforo $\left(\mathrm{P}_{2} \mathrm{O}_{5}\right)$, elemento essencial no desenvolvimento do sistema radicular, conforme resultados também obtidos por Souto et al. (2009). 
VIEIRA NETO, J. et al. Fertilizantes na produção de mudas...
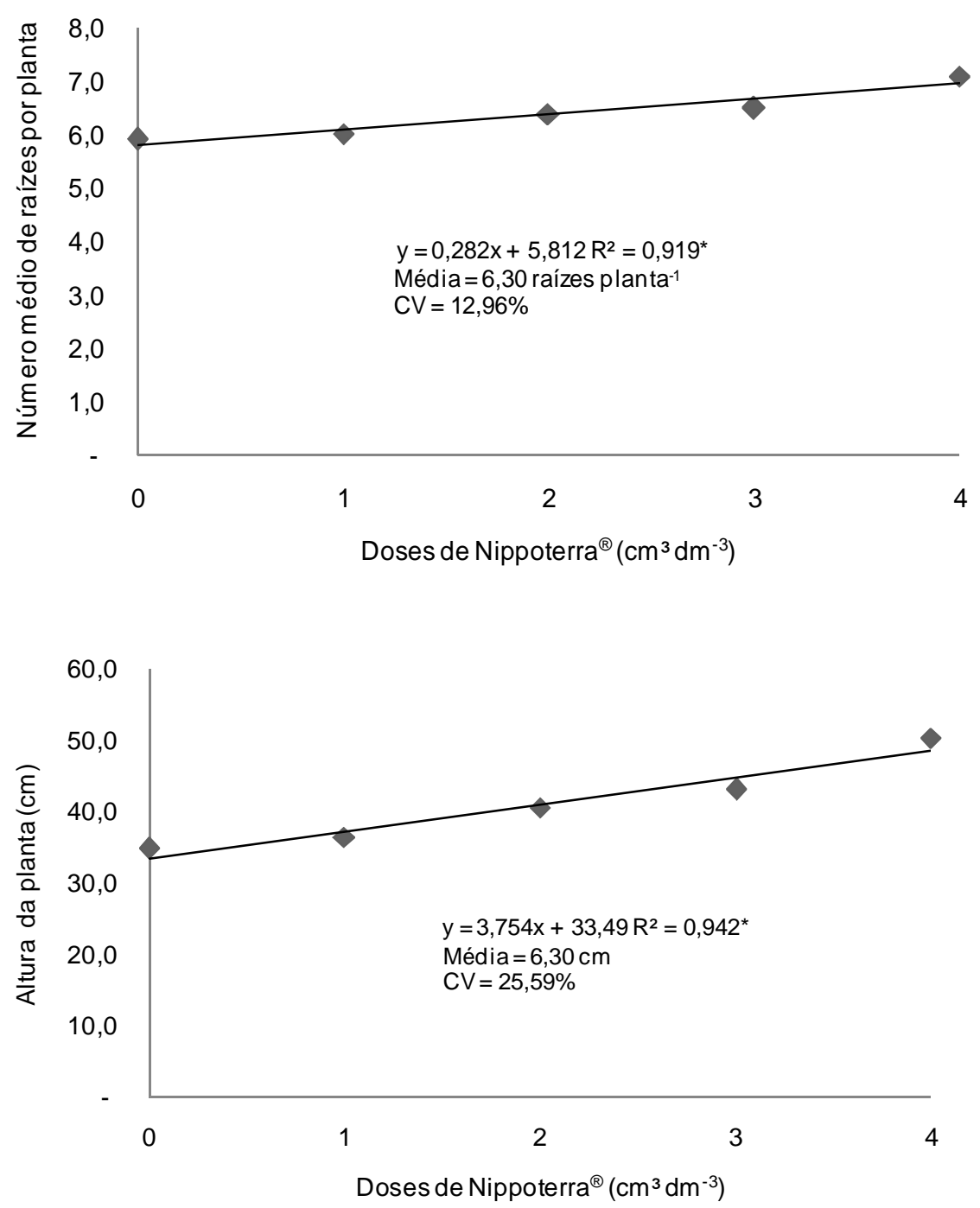

FIGURA 2 - Número médio de raízes por planta $(\mathrm{A})$ e altura da planta $(\mathrm{B})$ de mudas da oliveira 'Arbequina', após 120 dias da aplicação de diferentes doses de Nippoterra ${ }^{\circledR}$. EPAMIG, Maria da Fé - MG, 2009. * Significativo a $5 \%$ de probabilidade pelo teste F.

Todos os fertilizantes afetaram significativamente a massa seca da parte aérea e da raiz (Figuras $4 \mathrm{~A}$ e $4 \mathrm{~B}$ ) em relação à testemunha (sem fertilizante). Os maiores valores estimados para massa seca da parte aérea e massa seca da raiz foram obtidos com $4,0 \mathrm{~cm}^{3} \mathrm{dm}^{-3}$ de BrotaxMicro ${ }^{\circledR}$, ou seja, $4,01 \mathrm{~g} \mathrm{e} 1,48 \mathrm{~g}$, respectivamente. Ainda é possível observar que Nippoterra $^{\circledR}$ e Ativo $^{\circledR}$ apresentaram respostas crescentes para a massa da parte aérea e raiz até a dose de $2,96 \mathrm{~cm}^{3} \mathrm{dm}^{-3}$ (ponto de máximo). A partir desse ponto, houve um declínio das fitomassas, indicando uma provável saturação de resposta. Entretanto, não foram observados sintomas visuais de toxidez nas plantas ao aplicar os fertilizantes até a dosagem de $4 \mathrm{~cm}^{3} \mathrm{dm}^{-3}$.

\section{CONCLUSÕES}

Pelos resultados obtidos conclui-se que: a) Os fertilizantes Ativo ${ }^{\circledR}$, Brotax-Micro ${ }^{\circledR}$, Fert Bokashi $^{\circledR}$ e Nippoterra ${ }^{\circledR}$ tiveram desempenho favorável na etapa final de produção de mudas de oliveira; b) Todos fertilizantes testados, quando aplicados na dosagem de $4 \mathrm{~cm}^{3} \mathrm{dm}^{-3}$, proporcionaram mudas maiores e com sistema radicular mais desenvolvido, em relação à testemunha.

\section{AGRADECIMENTO}

Os autores agradecem à Fundação de Amparo a Pesquisa do Estado de Minas Gerais FAPEMIG pelo apoio financeiro na execução desse trabalho. 
VIEIRA NETO, J. et al. Fertilizantes na produção de mudas...

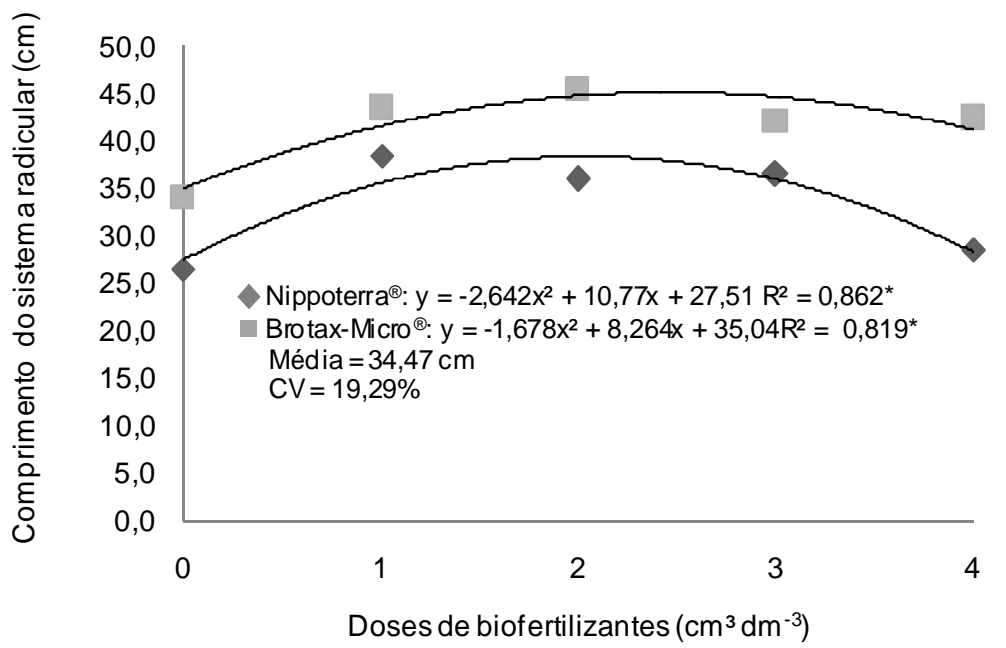

FIGURA 3 - Comprimento do sistema radicular de mudas da oliveira 'Arbequina', após 120 dias da aplicação de diferentes doses dos fertilizantes Nippoterra ${ }^{\circledR}$ e Brotax-Micro $^{\circledR}$. EPAMIG, Maria da Fé - MG, 2009. * Significativo a $5 \%$ de probabilidade pelo teste $\mathrm{F}$.

\section{CONFLITOS DE INTERESSE}

O presente trabalho não apresenta

conflitos de interesse que sejam do conhecimento dos autores, e não há relação de vínculo profissional ou de consultoria dos autores aos fabricantes dos produtos testados.

\section{REFERÊNCIAS}

1. AUED-PIMENTEL, S. et al. B. Determinação da diferença entre o valor real e o teórico do triglicerídeo ECN 42 para a detecção de adulteração em azeites de oliva comercializados no Brasil. Química Nova, v. 31, n. 1, p. 31-34, 2008.

2. COMPANHIA NACIONAL DE ABASTECIMENTO (CONAB). Indicadores da Agropecuária. Brasília, 2009. Disponível em: <http://www.conab.gov.br/conabweb/index.php?PAG=212>. Acesso em: 14 set. 2009.

3. CABALLERO, J. M.; DEL RIO, C. Métodos de multiplicación. In: BARRANCO, D.; FERNÁNDEZ-ESCOBAR, R.; RALLO, L. (Ed.). El cultivo del olivo. 2. ed. rev. y amp. Servilha: Consejería de Agricultura y Pesca de la Junta de Andalucíal Madrid: Mundi-Prensa, 1998. cap. 4, p. 89-113.

4. EMPRESA BRASILEIRA DE PESQUISA AGROPECUÁRIA (EMBRAPA). Ambiente de software NTIA, versão 4.2.2: manual do usuário - ferramental estatístico. Campinas: EMBRAPA/CNPTIA, 1997. 258 p.

5. FACHINELLO, J. C.; HOFFMANN, A.; NACHTIGAL, J. C. Propagação de plantas frutíferas. Brasília: EMBRAPA, 2005. $221 \mathrm{p}$.

6. FERnANDES, A. R.; CARVALHO, J. G. de; MELO, P. C. Efeito do fósforo e do zinco sobre o crescimento de mudas do cupuaçuzeiro (Theobroma grandiflorum Schum.). Cerne, v. 9, n. 2, p. 221-230, 2003.

7. LARCHER, W. Ecofisiologia vegetal. São Carlos: Rima, 2000. 531 p.

8. MALAVOLTA, E. Manual de nutrição mineral de plantas. São Paulo: Ceres, 2006. 631 p.

9. MESQUITA, D. L.; OLIVEIRA, A. F. de; MESQUITA, H. A. de. Aspectos econômicos da produção e comercialização do azeite de oliva e azeitona. Informe Agropecuário, v. 27, n. 231, p. 7-12, 2006.

10.NATALE W. et al. Resposta de mudas de goiabeira à aplicação de zinco. Revista Brasileira de Fruticultura, v. 24, n. 3 , p. 770-773, 2002.

11.OLIVEIRA, D. L. de. Multiplicação da oliveira através da enxertia, estaquia e ácido indolbutírico. 2007. 58 f. Dissertação (Mestrado em Fitotecnia) - Universidade Federal de Lavras, Lavras, 2007.

12.OLIVEIRA, A. F. et al. Influência do número de nós em estacas semilenhosas de oliveira (Olea europaea L.) no enraizamento sob câmara de nebulização. Ciência e Agrotecnologia, v. 27, n. 2, p. 332-338, 2003.

13. _. Pioneirismo marca pesquisa sobre oliveira em Minas Gerais. Informe Agropecuário, v. 30, n. edição especial. p. 109-117, 2009a.

14. Estaquia de oliveira em diferentes épocas, substratos e doses de AIB diluído em $\mathrm{NaOH}$ e álcool. Ciência e Agrotecnologia, v. 33, n. 1, p. 79-85, 2009b.

15.RALLO, L. Variedades de olivo en España: una aproximación cronológica. In: RALLO, L. et al. (Ed.). Variedades de olivo en España. Sevilla: Consejería de Agricultura y Pesca de la Junta de Andalucía/Madrid: Ministerio de Agricultura, Pesca y Alimentación/Mundi-Prensa, 1. ed., 2005. cap. 1, p. 17-44.

16.SÃO JOSÉ, A. R. et al. Formação de mudas de maracujazeiros. In: SÃO JOSÉ, A. R. (Ed). Maracujá: produção e mercado. Vitória da Conquista: UESB, 1994. p. 41-48.

17.SOARES, I.; LIMA, S. C.; CRISÓTOMO, S. A. Crescimento e composição mineral de mudas de gravioleira em resposta a doses de fósforo. Revista Ciência Agronômica, v. 38, n. 4, p. 343-349, 2007.

18.SOUTO, J. S. et al. Efeito da aplicação de fósforo no desenvolvimento de plantas de feijão Gandu (Cajanus cajan (L.) Millsp. Revista Verde, v. 4, n. 1, p. 135-140, 2009. 
VIEIRA NETO, J. et al. Fertilizantes na produção de mudas...
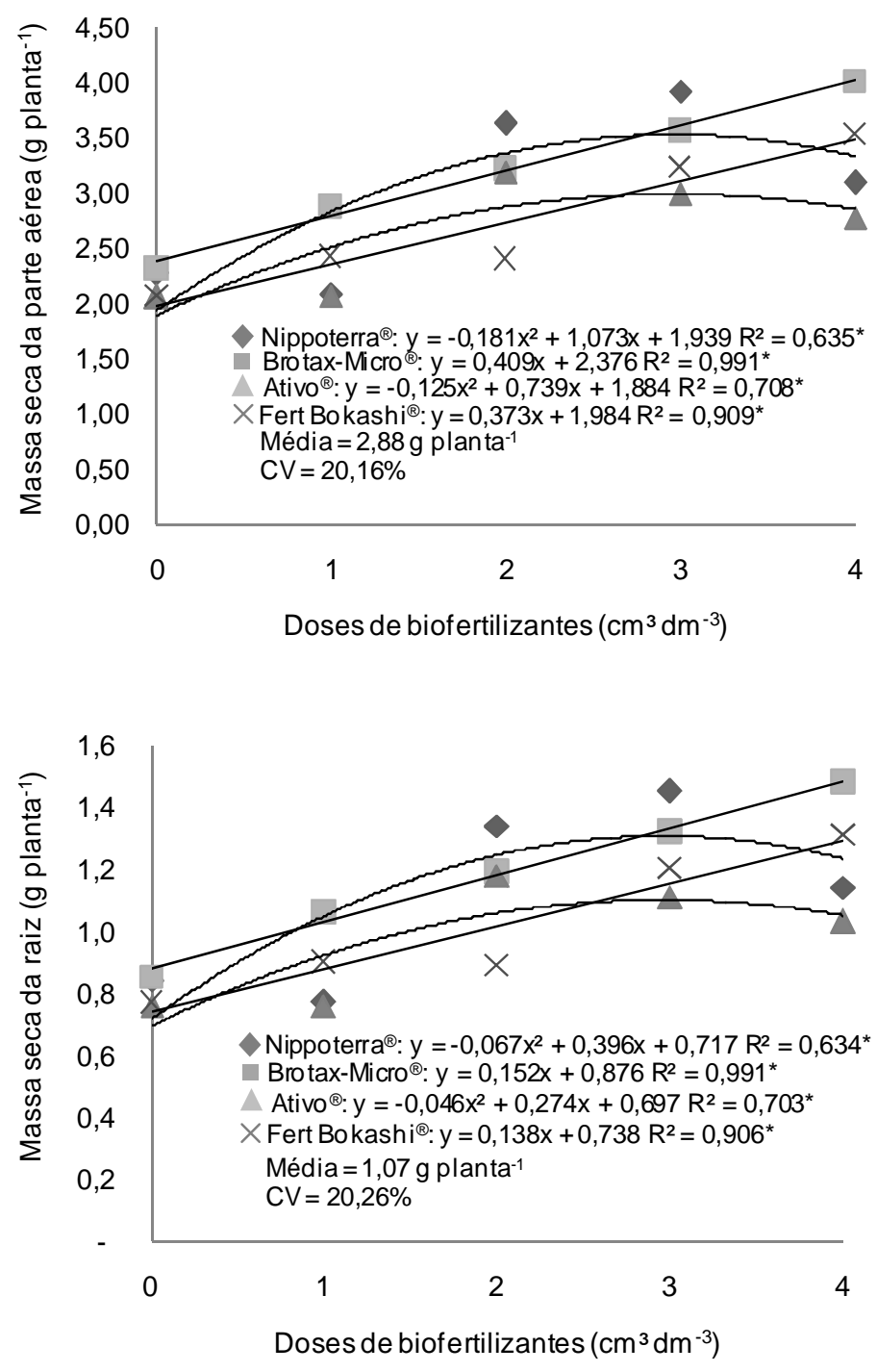

FIGURA 4 - Massa seca da parte aérea por planta (A) e massa seca da raiz por planta (B) de mudas da oliveira 'Arbequina' em função das doses de fertilizantes. EPAMIG, Maria da Fé - MG, 2009. * Significativo a $5 \%$ de probabilidade pelo teste $\mathrm{F}$.

19.SOUZA, F. X.; LIMA, R. N. de. Enraizamento de estacas de diferentes matrizes de cajazeira tratadas com ácido indolbutírico. Revista Ciência Agronômica, v. 36, n. 2, p. 189-194, 2005.

20.VIEIRA NETO, J. et al. Evaluation of formulations of leaf fertilizer at the finalization of the olive tree seedlings. In: EDITION OF THE FRUIT, NUT AND VEGETABLE PRODUCTION ENGINEERING SYMPOSIUM, 8., 2009, Concepción. Proceedings... Chillán: ProgapINIA, 2009. v. 1. p. 620-627. 
